GPO PRICE

CFSTI PRICE(S)

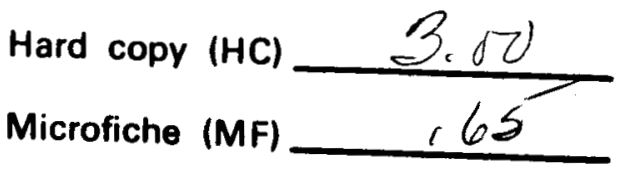

ff 653 July 65

\title{
EFFECT OF COULOMB COLLISIONS ON CONTRASTREAMING PLASMAS
}

\author{
B. Buti ${ }^{*}$
}

Theoretical Physics Division, National Physical Laboratory, New Delhi, India

and Laboratory for Theoretical Studies,

Goddard Space Flight Center, Greenbelt, Maryland

and

S. K. Trehan ${ }^{*}$

Department of Physics and Astrophysics, University of Delhi New Delhi, India

and Laboratory for Theoretical Studies, Goddard Space Flight Center, Greenbelt, Maryland

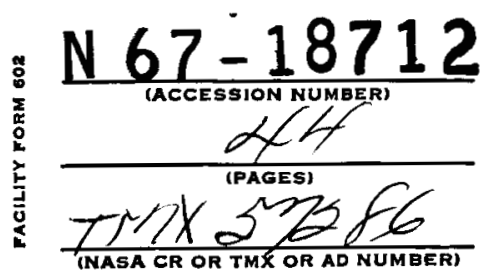

Present Adc

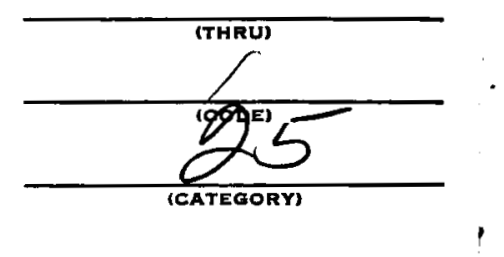

*National Atrunaulıs anu

Space Administration

coddard Space Flight Center

Lijoratory for Theoretical Stud

Greenbelt, Maryland 20771 


\section{ABSTRACT}

The instability of contrastreaming plasmas is investigated taking into account the Coulomb Collisions via the Fokker-Planck co-efficients in the Boltzmann equation. The dispersion relation is obtained on the assumption that the Coulomb collisions are weak and solved on the additional assumption that the phase velocity of the wave is much larger than the mean thermal velocity of the particles. It is found that while the temperature has the effect of increasing the maximum wavenumber $x_{c}$ (which for a cold plasma is equal to $(2)^{1 / 2}$ in units of $\omega_{p} / U$, where $\omega_{p}$ is the electron plasma frequency and $\bigcup_{m}$ is the streaming velocity) below which the plasma is unstable, the collisions have no effect on this wavenumber. However, the growth rate of maximum instability decreases (compared to its value for a cold plasma) on taking into account the thermal motions but increases when the collisions are taken into account. 


\section{INTRODUCTION}

The instability of longitudinal electron oscillations in a plasma where we have two streams of electrons moving with equal and opposite velocities has been discussed extensively by several authors $(1,2)$ when the thermal spread of the electrons as well as the collisions between the particles are ignored. It is well known that such a system is unstable for al1 wavenumbers $x\left(=k v / \omega_{p}\right.$, where $k$ is the wavenumber of the perturbation, $U$ the speed of the stream and $\omega_{p}$ the electron plasma frequency) which are less than a certain critical value $x_{c}=(2)^{1 / 2}$. Further the wavenumber for which the instability is maximum is known to be $x_{*}=(3)^{1 / 2} / 2$. Jackson (3) has considered the problem of two stream instability when the velocities of the two streams are smeared out by an equal amount and finds that $x_{c}$ increases on taking into account the spread of the distribution function of the electron streams.

Tidman and Weiss (4) calculated the effect of collisions on the two stream instability assuming zero thermal spread of the electrons and the ions. The collisions were taken into account by a simple relaxation model in which the electrons are taken to be scattered into a local Maxwellian distribution in a time $\mathcal{\tau}$. They found that collisions increased this instability. This result led them to suspect that the diffusion nature of the Coulomb thermalization of the electron streams is an essential feature of the problem and hence the more exact Fokker-Planck equation for Coulomb scattering should be used. It was later 
pointed out by May (b) that Timan and Veiss uid not carry out the calculations consistently as they neglected the equilibrium current density and the associated magnetic field which results due to the mass motion of the electrons. May considered two interpenetrating streams so that there is no current in equilibrium. He calculated the effect of collisions using the relaxation model of Gross and rrook (6) and Fondid that the unstable mode ceases to grow after a time of the order of one collision time. However, he had taken into account only the electron-electron collisions claiming that the electron-ion collisions are negligible due to the large ion-electron mass ratio. We will show here that the electron-ion collisions are actually comparable with the electronelectron collisions for the problem at hand.

Tidman (7) considered the effect of collisions on the instability of a stream of particles moving with a velocity $V_{m}$ (with no thermal spread) through cold ions at rest. He took into account the effect of Coulomb collisions using the Fokker-Planck equation and found that as the ordered streaming energy of the electrons is thermalized due to diffusion in velocity space, there results an enchanced Landau damping which competes with the growth of instability. He did not take into account the equilibrium current density which results in his model. It will be shown from our more rigorous calculations that weak collisions cannot even cancel the effect of thermal motions much less quenching the instability.

Comisar (8) and Buti and Jain (9) have studied the effect of weak Coulomb collisions on the damping of longitudinal and transverse 
plasma oscillations respectively using the Fokker-Planck equation in the form given by Rosenbluth et al (10). They find that to the first order in the collision frequency $\mathcal{V}_{\mathcal{C}}$, the electron-ion collisions dominate the electron-electron collisions in damping the plasma oscillations.

Following Comisar and Buti and Jain we consider the effect of Coulomb collisions on the stability of contrastreaming hot plasmas in a systematic manner. We assume that the collisions are not too frequent so that we can make an expansion in powers of $\left(\nu_{c} / \omega_{p}\right)$ and we calculate the collisional effects to the first order in the collision frequency. In the absence of collisions, the dispersion relation, including the thermal effects, is exactly solved numerically to determine the region of instability and the growth rate of maximum instability. When collisions are taken into account along with the thermal effects, then we use an iterative procedure to solve the dispersion relation and to study the effect uf collisions on the critical wavenumber and on the growth rate of maximum instability. It is, perhaps, in order to remark here that the Fokker-Planck equation is not strictly valid for an unstable plasma, particularly near the electron plasma frequency $(11,12)$. However, the motivation for the present undertaking is to clarify the contributions of the Erictional and the diffusion terms in the electron-electron and electron-ion collisions. We find that the contribution of the frictional term is dominant compared to that of the diffusion term in electron-ion collinions riereas in clectru-clectron collisions the two are of the same order. Further, the contribution of the 
electron-ion collisions is much larger than that of the electronelectron collisions. It may be reasonable to expect that the qualitative nature of these results will not be altered by using a more exact kinetic equation for an unstable plasma.

\section{II . GENERAL THEORY}

Let us consider two streams of hot unbounded plasmas in which the ions form only the neutralizing background but the electrons have non-relativistic streaming velocities. The equilibrium distribution functions, normalized to unity, for the electrons and the ions are assumed to have the form:

$$
f_{o a}=\left(2 \pi v_{a}^{2}\right)^{-3 / 2} e^{-\left(v-U_{a}\right)^{2} / 2 v_{a}^{2}}
$$

and

$$
f_{0 i}=\left(2 \pi v_{i}^{2}\right)^{-3 / 2} e^{-v^{2} / 2 v_{i}^{2}}
$$

where $V_{a}^{2}=\Theta_{a} / m$ and $V_{i}^{2}=\Theta_{i} / M ; \Theta_{a}$ and $\Theta_{i}$ denote the temperatures (in energy units) of the electrons and the ions respectively. The subscript a on the electron distribution function refers to either of the streams. The density of electrons on ions in each stream is taken to be $\mathrm{N}$. The distribution functions given by Eqs. (1) and (2) do not satisfy the Fokker-Planck equation:

$$
\frac{\partial f}{\partial t}+\underline{v} \cdot \nabla f+F_{m} \cdot \nabla_{m} f=-\frac{\partial}{\partial v} \cdot\left[\langle\Delta\rangle f-\frac{1}{2} \frac{\partial}{\partial v} \cdot\langle\Delta \Delta\rangle f\right],
$$

where $F$ is the force per unit mass and

$$
\langle\Delta\rangle=N \Gamma \sum_{\alpha=2, e}\left(1+\frac{m}{m_{\alpha}}\right) \frac{\partial}{\partial v} \int d v_{m}^{\prime} \frac{f\left(\underline{w}^{\prime}\right)}{\left|\underline{v}-\underline{w}^{\prime}\right|},
$$




$$
\langle\Delta \Delta\rangle=N \Gamma \sum_{\alpha=i, e} \frac{\partial^{2}}{\partial \underline{v} \partial \underline{v}} \int d \underline{v}^{\prime} f_{\alpha}\left(\underline{v}^{\prime}\right)\left|\underline{v}-\underline{v}^{\prime}\right|
$$

$$
\Gamma=\frac{4 \pi e^{4}}{m^{2}} \log \Lambda, \Lambda=4 \pi N \lambda_{D}^{3}
$$

where $\lambda_{D}$ is the Debye length. In fact we find that

$$
\frac{d U_{a}}{d t}=\frac{4 \nu_{c} U_{a}(t)}{(\pi)^{1 / 2}\left(U_{a} / v_{0}\right)^{3}}\left[2 \Omega\left(U_{a}^{2} / 2 v_{0}^{2}\right)+\frac{1}{4} \Omega\left(U_{a}^{2} / v_{0}^{2}\right)\right]
$$

where

$$
\Omega(x)=\int_{0}^{x} u^{2} e^{-u^{2}} d u,
$$

and the effective collision frequency is defined to be

$$
\nu_{c}=\left(\omega_{p} / \Lambda\right) \log \Lambda
$$

In writing Eq. (7) we have taken the electron temperatures of each stream to be the same and put $V_{a}^{2}=V_{0}^{2}$.

As we shall see later the effect of thermal motions enters the dispersion relation through the parameter $\left(k \lambda_{D}\right)^{2}=A x^{2}$ with $A=V_{0}^{2} / U^{2}$ and $x=k U / \omega p$.

Now for all situations of physical interest $\left(k \lambda_{D}\right)$ must be much

less than unity. Further we know that two-stream instability arises for values of $x$ of order unity. Thus the condition $k \lambda_{D} \ll /$ implies

$-7-$ 
that $A$ be less than unity for the unstable situations we are considering here. Since

$$
\Omega\left(u_{a}^{2} / v_{0}^{2}\right) \simeq \frac{1}{4}(\pi)^{1 / 2} \text { for } v_{0}^{2} / U_{a}^{2} \ll 1
$$

we can write

$$
\frac{d U_{a}}{d t}=\frac{9}{4} \nu_{c} U_{a}(t)\left(\frac{V_{0}}{U_{a}}\right)^{3},
$$

or in order of magnitude

$$
\frac{1}{\omega_{p} U} \frac{d U}{d t}=\frac{q}{4} \frac{\nu}{\omega_{p}}\left(\frac{V_{0}}{U}\right)^{3} .
$$

The quantity $\left(\mathcal{V}_{c} / \omega_{p}\right)$ is always much less than unity for physical situations of interest and we have seen that $\left(V_{0} / U_{a}\right)$ is also less than unity for the unstable situations under consideration here. Hence $U$ changes in time on a scale which is very much 1 arger than a plasma period. On the other hand we know that in the absence of collisions the two stream instability has e-folding time of the order of a plasma period. Thus for the problem at hand, the distribution function satisfies the Fokker-Planck equation to a good approximation and we may take $\bigcup_{\mathfrak{m}}$ in $\mathrm{Eq} .(1)$ to be constant. Moreover, the neglect of this term is justified on the ground that this is independent of $\boldsymbol{x}$ and thus would affect only the $k=0$ mode when we take the Fourier transform of the linearized equations in $\boldsymbol{x}$ - space; and we are not primarily concerned with this mode here. In a similar 
manner, it can be shown that the Fokker-Planck equation for the ions is also satisfied to an equally good approximation.

For longitudinal oscillations the linearized Fokker-Planck equation for the motion of electrons has the form:

$$
\frac{\partial f_{a}}{\partial t}+v \cdot \nabla f_{a}-\frac{N e}{m} E \cdot \nabla_{m} f_{o a}=\left(\frac{\partial f_{a}}{\partial t}\right)_{\text {coll. }},
$$

where the right hand side represents the effect of collisions.

Again the subscript a in Eq. (13) implies that this equation refers to either of the plasma streams, $a=1$ or 2 . The change in $f_{a}$ due to collisions results from collisions with the ions or electrons in either stream. Thus we can write

$$
\left(\frac{\partial f_{a}}{\partial t}\right)_{\text {coll. }}=\sum_{b}\left[\left(\frac{\partial f_{a}}{\partial t}\right)_{i b}+\left(\frac{\partial f_{a}}{\partial t}\right)_{e b}\right]
$$

where $a, b=1$ or 2 . The collision terms are given by

$$
\left(\frac{\partial f_{a}}{\partial t}\right)_{i b}=-\frac{\partial}{\partial v} \cdot\left(\langle\Delta\rangle_{0 i}^{b} f_{a}\right)+\frac{1}{2} \frac{\partial^{2}}{\partial v_{m} \partial \underline{w}}:\left(\langle\Delta \Delta\rangle_{o i}^{b} f_{a}\right)
$$

and

$$
\begin{aligned}
\left(\frac{\partial f_{a}}{\partial t}\right)_{e b} & =-\frac{\partial}{\partial v} \cdot\left(\langle\Delta\rangle_{o e}^{b} f_{a}+\langle\Delta\rangle_{e}^{b} f_{o a}\right) \\
& +\frac{1}{2} \frac{\partial^{2}}{\partial \underline{\partial} \partial \underline{m}}:\left(\langle\Delta \Delta\rangle_{o e}^{b} f_{a}+\langle\Delta \Delta\rangle_{e}^{b} f_{o a}\right),
\end{aligned}
$$

where

$$
\langle\Delta\rangle_{o i}^{b}=N \Gamma\left(1+\frac{m}{M}\right) \frac{\partial}{\partial v} \int \frac{f_{\Delta i}\left(\underline{w}^{\prime}\right) d v^{\prime}}{\left|\underline{m}-\underline{w}^{\prime}\right|},
$$




$$
\begin{aligned}
& \langle\Delta \Delta\rangle_{o i}^{\Delta}=N \Gamma \frac{\partial^{2}}{\partial \underline{v} \partial \underline{v}} \int f_{o i}\left(\underline{v}^{\prime}\right)\left|\underline{v}-\underline{v}^{\prime}\right| d \underline{v}^{\prime}, \\
& \langle\Delta\rangle_{o e}^{b}=2 N \Gamma \frac{\partial}{\partial \underline{v}} \int \frac{f_{0 b}\left(\underline{v}^{\prime}\right)}{\left|\underline{v}-\underline{v}^{\prime}\right|} d \underline{v}^{\prime}, \\
& \langle\Delta \Delta\rangle_{o e}^{b}=N \Gamma \frac{\partial^{2}}{\partial v \partial v} \int f_{0 b}\left(\underline{w}^{\prime}\right)\left|\underline{v}-\underline{v}^{\prime}\right| d \underline{w}^{\prime} .
\end{aligned}
$$

The expressions for $\langle\Delta\rangle_{e}^{b}$ and $\langle\Delta \Delta\rangle_{e}^{b}$ can be written down from Eq. (19) and (20) on replacing $f_{0 b}$ by $f_{b}$. The electric field $\underset{m}{E}$ in Eq. (13) is governed by the Poisson equation:

$$
\nabla \cdot E=-4 \pi e \sum_{a} \int f_{a}(x, y, t) d \underline{m} .
$$

Equations (13) and (21) from the characteristic value problem which we shall solve by the Fourier-Laplace transform method. We thus define

$$
f_{k}^{a}(v, s)=\int_{0}^{\infty} d t \int d x e^{-s t-i k d x} f_{a}(x, v, t),
$$

and

$$
E_{m k}(s)=\int_{0}^{\infty} d t \int d x e^{-s t-i \underset{w}{k} \cdot x_{m}} \underset{m}{E}(x, t),
$$

with Res>ofor the integrals to be convergent. The Fourier-Laplace transforms of Eqs. (13) and (21) are:

$$
\begin{aligned}
\Delta f_{k}^{a}(r, s) & -g_{k}^{a}(\underline{w})+i k \cdot f_{m}^{a}(v, s) \\
-\frac{N e}{m} E_{m}(s) \cdot \frac{\partial f_{0 a}}{\partial v}= & \left(\frac{\partial f_{k}^{a}}{\partial t}\right)_{\text {coll }} . \\
& -10-
\end{aligned}
$$


and

$$
E_{m k}(s)=4 \pi i e \frac{k}{k^{2}} \sum_{a} \int f_{k}^{a}(k, s) d v,
$$

where $g_{k}^{a}(\underline{v})$ is the Fourier transform of the initial distribution function ie.,

$$
g_{k}^{a}(v)=\int d x e^{-i k \cdot x} f_{a}(x, v, 0) \text {. }
$$

In order to solve the characteristic value problem posed by Ens. (24) and (25), it is now convenient to take the Fourier-transform in velocity space; we thus define

$$
F_{a}(k, \sigma, s)=\int f_{k}^{a}(x, s) e^{-i \sigma \cdot v} d v
$$

Equation (24) now takes the form

$$
\begin{aligned}
& \left(A-\frac{k}{m} \cdot \frac{\partial}{\partial \sigma}\right) F_{a}(k, \sigma, s)-G_{a}(k, \sigma) \\
& -\frac{i N e}{m} \sigma \cdot E_{k} \exp \left\{-i \sigma \cdot U_{m}-\frac{1}{2} \sigma^{2} V_{0}^{2}\right\}=\sum_{b}\left[\left(\frac{\partial F_{a}}{\partial t}\right)_{i b}+\left(\frac{\partial F_{a}}{\partial t}\right)_{e b}\right] .
\end{aligned}
$$

In order to simplify the collision terms, we first observe that the Fourier-Laplace transform of Eq. (15) leads to

$$
\begin{aligned}
\left(\frac{\partial F_{a}}{\partial t}\right)_{i b}=\int d v_{m}^{\prime} & e^{-i \sigma \cdot v^{\prime}}\left[-i \sigma \cdot\langle\Delta\rangle_{o i}^{b}\right. \\
& \left.-\frac{1}{2} \sigma \sigma:\langle\Delta \Delta \Delta\rangle_{o i}^{b}\right] f_{k}^{a}\left(x_{m}, v^{\prime}, s\right) .
\end{aligned}
$$

On using the identies

and

$$
\frac{1}{\left|\underline{m}-\underline{w}^{\prime}\right|}=\frac{1}{2 \pi^{2}} \int d \xi e^{i \xi \cdot\left(\underline{m}-\underline{w}^{\prime}\right)} \frac{1}{\xi^{2}}
$$

$$
\left|\underline{m}-\underline{m}^{\prime}\right|=-\frac{1}{\pi^{2}} \int d \xi_{-11} e^{i \xi \cdot\left(v_{m}-x^{\prime}\right)} \frac{1}{\xi^{4}},
$$


we find that

$$
\langle\Delta\rangle_{o i}^{b}=N \Gamma \frac{1}{2 \pi^{2}}\left(1+\frac{m}{M}\right) \int d \xi \frac{i \xi}{\xi^{2}} \exp \left\{i \xi \cdot v_{m}-\frac{1}{2} V_{i}^{2} \xi^{2}\right\}
$$

$(30 a)$

and

$$
\langle\Delta \Delta\rangle_{o i}^{b}=\frac{N \Gamma}{\pi^{2}} \int d \xi \frac{\xi \xi}{\xi_{m}^{4}} \exp \left\{i \xi_{m} \cdot v-\frac{1}{2} V_{i}^{2} \xi^{2}\right\} \text {. }
$$

On using the foregoing results in Eq. (29) we obtain

$$
\left(\frac{\partial F_{a}}{\partial t}\right)_{i b}=N \Gamma \int d \eta K_{1}^{b}(\sigma, \eta) F_{a}(k, \eta, s) \text {, }
$$

$$
\begin{aligned}
& K_{1}(\sigma, \eta)=\frac{1}{2 \pi^{2}}\left[\left(1+\frac{m}{M}\right) \frac{\sigma \cdot(\sigma-\eta)}{|\sigma-\eta|^{2}}\right. \\
& \left.-\frac{\{\sigma \cdot(\sigma-\eta)\}^{2}}{|\sigma-\eta|^{2}}\right] \exp \left\{-\frac{1}{2} v_{i}^{2}(\sigma-\eta)^{2}\right\} \text {. } \\
& \left(\frac{\partial F_{a}}{\partial t}\right)_{e b}=N \Gamma \int d \eta\left[K_{2}^{b} F_{a}(k, \eta, p)+K_{3}^{a} F_{b}(k, \eta, \nu)\right], \\
& \text { where } \\
& K_{2}^{a}(\sigma, \eta)=\frac{1}{\pi^{2}}\left[\frac{\sigma \cdot(\sigma-\eta)}{|\sigma-\eta|^{2}}-\frac{1}{2} \frac{\{\sigma \cdot(\sigma-\eta)\}^{2}}{|\sigma-\eta|^{4}}\right] \\
& x \exp \left\{-\frac{1}{2} v_{0}^{2}(\sigma-\eta)^{2}-i u_{a} \cdot(\sigma-\eta)\right\} \\
& K_{3}^{a}(\sigma, \eta)=\frac{1}{\pi^{2}}\left[\frac{\sigma \cdot \eta}{\eta^{2}}-\frac{1}{2} \frac{(\sigma \cdot \eta)^{2}}{\eta^{4}}\right] \\
& x \exp \left\{-\frac{1}{2} v_{c}^{2}(\sigma-\eta)^{2}-i u_{m} \cdot(\sigma-\eta \eta q)\right\} \text {. }
\end{aligned}
$$

$-12-$ 
We shall now take $k$ to be along the $z$-axis and also assume that $U_{m}=U_{a} e_{z}$ and further we set $N \Gamma=V_{0}^{4} / L$, where $L$ represents the mean free path of the particles between collisions. Eq. (28) now reduces to

$$
\begin{aligned}
& \left(\frac{\Delta}{k}-\frac{\partial}{\partial \sigma_{z}}\right) F_{a}(k, \sigma, s)-\frac{1}{k} G_{a}(k, \sigma) \\
& -\frac{i N_{e}}{m k} \sigma \cdot F_{m k} \exp \left\{-\frac{1}{2} \sigma^{2} v_{0}^{2}-i \sigma_{z} U_{a}\right\}=\frac{V_{0}^{4}}{k L} \sum_{b} \int d \eta\left[\left\{K_{1}^{b}(\sigma, \eta)\right.\right. \\
& \left.\left.+K_{2}^{b}(\sigma, \eta)\right\} F_{a}(k, \eta, s)+K_{3}^{a}(\sigma, \eta) F_{b}(k, \eta, s)\right] .
\end{aligned}
$$

Equation (36) can be readily integrated to give

$$
\begin{aligned}
& F_{a}(k, \sigma, s)=e^{s \sigma_{z} / k}\left[-\frac{1}{k} \int_{\infty}^{\sigma_{z}} e^{-s \sigma_{z}^{\prime} / k} G_{a}\left(k, \sigma_{m}^{\prime}\right) d \sigma_{z}^{\prime}\right. \\
& -\frac{i N_{e}}{m k} E_{k}(s) \cdot \int_{\infty}^{\sigma_{z}} \sigma^{\prime} \exp \left\{-\frac{1}{2} \sigma^{2} v_{a}^{2}-i \sigma_{z}^{\prime} U_{a}-s \sigma_{z}^{\prime} / k\right\} d \sigma_{z}^{\prime} \\
& -\frac{v_{0}^{4}}{k L} \sum_{b} \int_{\infty}^{\sigma_{z}} d \sigma_{z}^{\prime} \int d^{3} \eta e^{-s \sigma_{z}^{\prime} / k}\left[\left\{K_{1}^{b}\left(\sigma_{m}^{\prime}, \eta\right)+K_{2}^{b}\left(\sigma_{m}^{\prime}, \eta\right)\right\} F_{a}(k, \eta, s)\right. \\
& \left.\quad+K_{3}^{a}\left(\sigma^{\prime}, \eta\right) F_{b}(k, \eta, s)\right], \\
& \text { where } \sigma_{m}^{\prime}=\left(\sigma_{x}, \sigma_{y}, \sigma_{z}^{\prime}\right) .
\end{aligned}
$$

We shall now assume that the collisions are weak so that

$k L \gg 1$. Under this assumption, to the lowest order, Eq. (37)

gives

$$
\begin{gathered}
F_{a}(k, \sigma, s)=e^{s \sigma_{z} / k}\left[Q_{a}(k, \sigma)-\frac{i N_{e}}{m k} E_{k} \cdot P_{a}(k, \sigma)\right], \\
-13-
\end{gathered}
$$




$$
\begin{gathered}
Q_{a}(k, \sigma)=-\frac{1}{k} \int_{\infty}^{\sigma_{z}} d \sigma_{z}^{\prime} G_{a}\left(k, \sigma_{m}^{\prime}\right) e^{-s \sigma_{z}^{\prime} / k}, \\
P_{m}(k, \sigma)=\int_{\infty}^{\sigma_{z}} d \sigma_{z}^{\prime} \sigma_{m}^{\prime} \exp \left\{-s \sigma_{z}^{\prime} / k-\frac{1}{2} \sigma^{\prime} v_{0}^{2}-i \sigma_{z}^{\prime} U_{a}\right\} .
\end{gathered}
$$

We now substitute the zero-order solution given by Eq. (38) into

$$
\begin{aligned}
& F_{a}(k, \sigma, \Delta)=e^{\Delta \sigma_{z} / k} \llbracket Q_{a}(k, \sigma)+\frac{v_{0}^{4}}{k L} \sum_{\xi} \int_{\sigma_{z}}^{\infty} d \sigma_{z}^{\prime} \int d \eta e^{-\Delta\left(\sigma_{z}^{\prime}-\eta_{z}\right) / k} \\
& \times\left\{\left[K_{1}^{b}\left(\sigma^{\prime}, \eta\right)+K_{2}^{b}\left(\sigma^{\prime}, \eta\right)\right] Q_{a}(k, \eta)+K_{3}^{q}\left(\sigma^{\prime}, q\right) Q_{b}(k, \eta)\right\}(41) \\
& -\frac{i N e}{m k} E_{k}(\Delta) \cdot\left\{P(k, \sigma)+\frac{v_{0}^{4}}{k L} \sum_{k} \int_{\sigma_{z}}^{\infty} d \sigma_{z}^{\prime} \int d \eta e^{-\rho\left(\sigma_{z}^{\prime}-\eta_{z}\right) / k}\right. \\
& \left.\left.\left.\times\left[\left\{K_{1}^{b}\left(\sigma_{m}^{\prime}, \eta\right)+K_{2}^{b}\left(\sigma^{\prime}, \eta\right)\right\} P_{a}(k, \eta)+K_{3}^{a}\left(\sigma^{\prime}, \eta\right) P_{b}(k, \eta)\right]\right\}\right]\right] \text { From Eq. (25) we. have } \\
& E_{k}(s)=\frac{4 \pi i e}{k} \sum_{a} F_{a}(k, 0, s) \text {. }
\end{aligned}
$$

On substituting for $F_{a}$ in accordance with $\mathrm{Eq}$. (41) into $\mathrm{Eq} .(42)$, we readily obtain

$$
E_{k}(s)=\frac{\Phi(k, s)}{\Psi(k, s)},
$$

where

$$
\begin{aligned}
& \Phi(k, s)=\frac{4 \pi i e}{k} \sum_{a}\left[Q_{a}(k, 0)\right. \\
& +\frac{V_{0}^{4}}{k L} \sum_{b} \int_{0}^{\infty} d \sigma_{z} \int_{d \eta} \exp \left\{-s\left(\sigma_{z}-\eta_{z}\right) / k\right\} \times\left\{\left(K_{1}^{b}\left(\sigma_{z}, \eta\right)\right.\right. \\
& \left.\left.\left.\left.+K_{2}^{b}\left(\sigma_{z}, \not\right)\right) Q_{a}(k, \not)+K_{3}^{a}\left(\sigma_{z}, \not{z}\right) Q_{b}(k, \not)\right)\right\}\right]
\end{aligned}
$$




$$
\begin{aligned}
& \Psi(k, s)=1-\frac{\omega_{p}^{2}}{k^{2}} \sum_{a}\left[\left[P_{a z}(k, 0)+\frac{v_{0}^{4}}{k L} \sum_{b} \int_{0}^{\infty} d \sigma_{z} \int d \eta\right.\right. \\
& x e^{-\frac{\partial}{k}\left(\sigma_{z}-\eta_{z}\right)}\left[\left\{K_{1}^{b}\left(\sigma_{z}, \eta\right)+K_{2}^{b}\left(\sigma_{z}, \eta_{1}\right)\right\} P_{a z}\left(k, \eta_{n}\right)\right. \\
& \left.\left.\left.+K_{3}^{a}\left(\sigma_{z}, y_{1}\right) P_{b z}(k, \eta)\right)\right]\right] \text {. }
\end{aligned}
$$

We may note here that $\Phi(k, s)$ depends only on the initial perturbation. If we consider only those perturbations for which $\Phi(k, s)$ is analytic in the complex s-plane, then for the Laplace inversion of $\mathrm{Eq}$. ( 43$)$, we have to consider only the zeros of $\Psi(\mathrm{k}, \mathrm{s})$ which are given by

$$
\Psi(k, s)=0 \text {. }
$$

This gives us the desired dispersion relation. It is now convenient to define the effective collision frequency as $\nu_{c}=V_{0} / L$. The dispersion relation then reads

$$
\begin{aligned}
& 1=\frac{\omega_{p}^{2}}{k^{2}} \sum_{a}\left[P_{a z}(k, 0)+\frac{\nu_{c} v_{0}^{3}}{k} \sum_{b} \int_{0}^{\infty} d \sigma_{z} \int d \eta\right. \\
& x e^{-s\left(\sigma_{z}-\eta z\right) / k}\left[\left\{K_{1}^{b}\left(\sigma_{z}, \eta\right)+K_{2}^{b}\left(\sigma_{z}, \eta\right)\right\} P_{a z}(k, \eta)\right. \\
& \left.\left.\left.\quad+K_{3}^{a}\left(\sigma_{z}, \eta\right) P_{b z}(k, \eta)\right]\right]\right] .
\end{aligned}
$$

III. EVALUATION OF THE INTEGRALS

We first need to evaluate

$$
P_{a z}\left(k, \eta_{n}\right)=\int_{\infty}^{\eta_{z}} d \eta_{z}^{\prime} \eta_{z}^{\prime} \exp \left(-\frac{p}{k} \eta_{z}^{\prime}-\frac{1}{2} v_{0}^{2} \eta^{\prime 2}-i \eta_{z}^{\prime} U_{a}\right),
$$


where $\eta^{\prime}=\left(\eta_{x}, \eta_{y}, \eta_{z}^{\prime}\right)$. It is convenient to write this as

$$
P_{a z}(k, \eta)=-\exp \left(\alpha_{a}^{2}-\frac{1}{2} v_{0}^{2} \eta_{1}^{2}\right) \int_{\eta_{z}}^{\infty} d \eta_{z}^{\prime} \eta_{z} \exp -\left(\alpha_{a}+V_{0} \eta_{z}^{\prime} /(2)^{1 / 2}\right)^{2} \text {, }
$$

where

$$
\eta_{L}^{2}=\eta^{2}-\eta_{z}^{2} \text { and } \alpha_{a}=\left(s / k+i U_{a}\right)\left\{V_{0}(2)^{1 / 2}\right\} \text {. }
$$

The integral which occurs in Eq. (49) can be expressed in terms of the error function and we find that

$$
P_{a z}(k, \eta)=-\frac{1}{v_{0}^{2}} \exp \left(\alpha_{a}^{2}-\frac{1}{2} v_{0}^{2} \eta_{\perp}^{2}-\eta_{+}^{2}\right)\left[1-2 \alpha_{a} e^{\eta_{+}^{2}} E r f\left(\eta_{+}\right)\right] \text {, }
$$

where $\eta_{+}=\alpha_{a}+\nu_{0} \eta_{z} /(2)^{1 / 2}$ and

$$
\operatorname{Erf}(x)=\int_{x}^{\infty} e^{-y^{2}} d y \text {, }
$$

which for large values of the argument has the asymptotic expansion

$$
\text { Er }(x)=\frac{e^{-x^{2}}}{2 x}\left[1-\frac{1}{2 x^{2}}+\frac{3}{2^{2} x^{4}}-\frac{1.3 .5}{2^{3} x^{6}}+\cdots\right] \text {. }
$$

On making the legitimate assumption that the phase velocity of the wave is much larger than the mean thermal speed i.e. $\Delta / k v_{0} \gg 1$, we obtain:

$$
P_{a z}(k, 0)=\frac{k^{2}}{\left(\omega-k v_{a}\right)^{2}}\left[1+\frac{3 k^{2} v_{0}^{2}}{\left(\omega-k v_{a}\right)^{2}}+\cdots\right] \text {, }
$$

where we have put $\Delta=-i \omega f$ or the sake of convenience.

$$
I_{b}^{a}=\int_{0}^{\infty} d \sigma_{z} \int d \eta e^{-s\left(\sigma_{z}-\eta_{z}\right) / k} K_{1}^{b}\left(\sigma_{z}, \eta\right) P_{a z}\left(k, \eta_{2}\right) .
$$


We may note that $K_{1}^{b}$ is independent of $U$ and as such of $b$.

we can, therefore, write $I_{1}^{a}=I_{2}^{b}=I_{b}^{a}, \quad b=1,2$. In order

to compare the relative orders of the diffusion term and the frictional term, we split up Eq. (55) as follows:

$$
I_{1 f, d}^{a}=\int_{0}^{\infty} d \sigma_{z} \int d \eta e^{-s\left(\sigma_{z}-\eta_{z}\right) / k} K_{1 f, d}\left(\sigma_{z}, y\right) P_{a z}(k, y),
$$

where $I_{d f}^{a}$ and $I_{1 d}^{a}$ represent the contribution of the frictional and the diffusion terms respectively to the electron-ion collisions. Further

$$
K_{1 f}(\sigma, \eta)=\frac{1}{2 \pi^{2}}\left(1+\frac{m}{M}\right) \frac{\sigma\left(\sigma-\eta \eta_{z}\right)}{\left|\sigma e_{z}-\eta\right|^{2}} \exp \left\{-\frac{1}{2} v_{c}^{2}\left(\sigma e_{z}-\eta\right)^{2}\right\},{ }_{(57)}
$$

and

$$
K_{1 d}(\sigma, \eta)=-\frac{1}{2 \pi^{2}} \frac{\left\{\sigma\left(\sigma-\eta_{z}\right)\right\}^{2}}{\left|\sigma_{m z}-\eta\right|^{4}} \exp \left\{-\frac{1}{2} V_{i}^{2}\left(\sigma e_{z}-\eta\right)^{2}\right\} .
$$

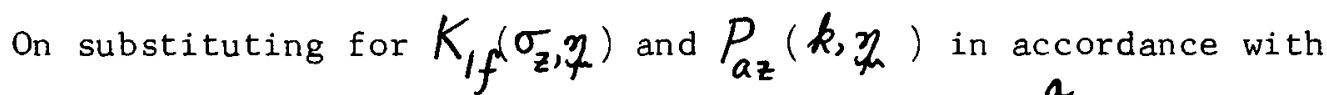
Eq. (57) and (51) into Eq. (56), we obtain for $I_{1 f}^{a}$ :

$$
\begin{aligned}
I_{1 f}^{a}= & -\frac{e^{\alpha_{a}^{2}}}{2 \pi^{2} v_{0}^{2}} \int_{0}^{\infty} d \sigma e^{-\Delta \sigma / k} \int d \eta_{m} \operatorname{sxp}\left\{\frac{\Delta}{k} \eta_{z}-\frac{1}{2} v_{c}^{2} \eta_{z}^{2}-\frac{1}{2} r_{c}^{2}\left(\sigma_{m}-\eta\right)^{2}\right\} \\
& \times\left(e^{-\eta_{+}^{2}}-2 \alpha_{a} E_{z} f\left(\eta_{+}\right)\right)\left[(1+m / M) \frac{\sigma\left(\sigma-\eta_{z}\right)}{\left(\sigma e_{z}-\eta\right)^{2}}\right] .
\end{aligned}
$$

It is now convenient to introduce the variable $\xi_{m}=\sigma_{m z}-\not 2$.

The integral $I_{1 f}^{a} \quad$ can then be written as:

$$
\begin{aligned}
I_{1 f}^{a}= & -\frac{e_{a}^{\alpha_{a}^{2}}}{2 \pi^{2} v_{0}^{2}} \int_{0}^{\infty} d \sigma \int d \xi \exp \left\{-\frac{1}{2}\left(v_{0}^{2}+v_{i}^{2}\right) \xi_{\perp}^{2}-\frac{1}{2} v_{i}^{2} \xi_{z}^{2}-\frac{\Delta}{k} \xi_{z}\right\} \\
& \times\left\{e^{-\xi_{+}^{2}}-2 \alpha_{a} \operatorname{Exf}_{j}\left(\xi_{+}\right)\right\}\left[\left(1+\frac{m}{M}\right) \frac{\sigma \xi_{z}}{\xi^{2}}\right],
\end{aligned}
$$

$-17-$ 
Where now $\xi_{+}=\alpha_{a}+V_{\Delta}\left(\sigma_{-} \xi_{z}\right) /(2)^{1 / 2}$. Let us introduce a system of cylinderical polar coordinates $\left(\xi_{\mathcal{L}}, \theta, \xi_{z}\right)$; Eq. (60) then reduces to

$$
\begin{aligned}
& I_{\text {If }}^{a}=-\frac{e^{\alpha_{a}^{2}}}{\pi V_{0}^{2}} \int_{0}^{\infty} d \sigma \int_{-\infty}^{\infty} d \xi_{z} \exp \left(-\frac{\Delta}{k} \xi_{z}-\frac{1}{2} v_{i}^{2} \xi_{z}^{2}\right) \\
& \times\left\{e^{-\xi_{+}^{2}}-2 \alpha_{a} \operatorname{Erf}\left(\xi_{+}\right)\right\} S_{/ f}^{a} \text {, }
\end{aligned}
$$

where $S_{\text {If }}^{a}$ is defined to be

$$
S_{\text {If }}^{\text {If }}=\int_{0}^{\infty} d \xi_{\perp} \xi_{\perp} e^{-\frac{1}{2}\left(v_{0}^{2}+v_{i}^{2}\right) \xi_{\perp}^{2}}\left(1+\frac{m}{M}\right) \frac{\sigma \xi_{z}}{\xi_{\perp}^{2}+\xi_{z}^{2}} .
$$

If we now put $\xi_{\perp}^{2}=t \xi_{z}^{2}$, this integral can be written as

$$
S_{\text {If }}^{a}=\frac{1}{2} \int_{1}^{\infty} \frac{d \nu}{\nu}\left(1+\frac{m}{M}\right) \sigma \xi_{z} \exp \left\{(1-\nu) b^{2} \xi_{z}^{2}\right\}, \text { (63) }
$$

where $b^{2}=\left(V_{0}^{2}+V_{i}^{2}\right) / 2$. The integral $I_{1 f}^{a}$ then takes the form

$$
\begin{aligned}
I_{I_{f}}^{a}= & -\frac{e^{\alpha_{a}^{2}}}{2 \pi v_{0}^{2}} \int_{1}^{\infty} \frac{d \nu}{\nu} \int_{0}^{\infty} d \sigma \int_{-\infty}^{\infty} d \xi_{z} \exp \left(-\frac{\alpha}{k} \xi_{z}+\frac{1}{2} v_{0}^{2} \xi_{z}^{2}-\nu b^{2} \xi_{z}^{2}-\xi_{+}^{2}\right) \\
& \times\left(1+\frac{m}{M}\right) \sigma \xi_{z}\left[1-2 \alpha_{a} e^{\xi_{+}^{2}} \operatorname{Erf}\left(\xi_{+}\right)\right] .
\end{aligned}
$$

Again if we assume that $\xi_{+} \gg 11$, we can use the asymptotic expansion of error function and after some reductions we find

$$
I_{1 f}^{a}=-\frac{e^{\alpha_{a}^{2}}}{2 \pi v_{0}^{2}} \int_{1}^{\infty} \frac{d \nu}{\nu} \int_{0}^{\infty} d \sigma \frac{1}{b} \exp \left\{-\frac{1}{2} \beta^{2}+\frac{1}{4 \nu b^{2}}\left(v_{0}^{2} \sigma+i U_{a}\right)^{2}\right\} S_{2 f}^{a},(65)
$$

$-18-$ 
where

$$
\begin{aligned}
S_{2 f}^{a} & =\int_{-\infty}^{\infty} d x \exp \left\{-\nu\left[x-\frac{1}{2 \nu b}\left(v_{0}^{2} \sigma+i v_{a}\right)\right]^{2}\right\} m \mu \frac{\sigma x}{b} \\
& \times\left[1-\frac{a_{a}}{\beta-q x}+\frac{a_{a}}{(\beta-q x)^{3}}-\frac{3 a_{a}}{(\beta-q x)^{5}}+\frac{15 a_{a}}{(\beta-q x)^{7}}-\cdots\right],
\end{aligned}
$$

and we have put

$$
\begin{gathered}
\beta=\left(\frac{\alpha}{k}+i v_{a}+v_{0}^{2} \sigma\right) / v_{0}, q=v_{0} / b, a_{a}=(2)^{1 / 2} \alpha_{a}, \\
\mu=(m+M) / m M .
\end{gathered}
$$

We now observe that $\beta$ is usually much larger than $q$, being of the order of the phase velocity to the mean thermal velocity.

We can, therefore, carry out the expansions of the quantities appearing in the denominators in $S_{2 f}^{a}$. The resulting integrations are then elementary. We obtain after some straightforward calculations:

$$
\begin{aligned}
S_{2 f}^{a} & =\sqrt{\frac{\pi}{\gamma}} \frac{m \mu \sigma}{2 \nu b^{2}}\left[( v _ { 0 } ^ { 2 } \sigma + i U _ { a } ) \left\{1-a_{a}\left(\frac{1}{\gamma}-\frac{A_{2}}{\gamma^{3}}\right.\right.\right. \\
+\frac{3 A_{2}^{2}}{\gamma^{5}} & )\}-\frac{a_{a} v_{0}}{\gamma^{2}}\left\{1-\frac{3 A_{2}}{\gamma^{2}}+\frac{15}{\gamma^{4}} A_{2}^{2}\right\}\right],
\end{aligned}
$$

where we have retained terms up to $\gamma^{-6}$ only, and

$$
A_{2}=1-v_{0}^{2} / 2 b^{2} \nu, \quad \gamma^{2}=\beta-\frac{q}{2 \nu b}\left(v_{0}^{2} \sigma+i v_{a}\right) \text {. }
$$

On substituting the foregoing results in equation (65) and after some reductions we find

$$
I_{1 f}^{a}=-\frac{1}{2(\pi)^{1 / 2} v_{0}^{3} b} \int_{1}^{\infty} \frac{d \nu}{\nu^{3 / 2}} e^{-U_{a}^{2} / 4 \nu b^{2}} S_{3 f}^{a}
$$

where

$-19-$ 


$$
\begin{aligned}
& S_{3 f}^{a}=\frac{m \mu}{2 \nu b^{2}} \int_{0}^{\infty} d x x \exp \left\{-\left(A_{1} x+\frac{1}{2} A_{2} x^{2}\right)\right\}\left[\left(x+\frac{i U_{a}}{V_{0}}\right)\right. \\
& \quad \times\left\{1-\frac{a_{a}}{A_{1}+A_{2} x}\left[1-\frac{A_{2}}{\left(A_{1}+A_{2} x\right)^{2}}+\frac{3\left(1-A_{2} q^{2} / 2 \nu\right)}{\left(A_{1}+A_{2} x\right)^{4}}\right]\right\} \\
& \text { and } \left.-\frac{a_{a}}{\left(A_{1}+A_{2} x\right)^{2}}\left\{1-\frac{3 A_{2}}{\left(A_{1}+A_{2} x\right)^{2}}+\frac{15 A_{2}^{2}}{\left(A_{1}+A_{2} x\right)^{4}}\right\}\right], \\
& A_{1}=\frac{1}{V_{0}}\left(\frac{\hat{D}}{k}+i U_{a}\right)-\frac{i V_{0} U_{a}}{2 \nu b^{2}} .
\end{aligned}
$$

The various integrals occuring in Eq. (71) can be evaluated in terms of the error function Er $\left(y_{*}\right)$, where $y_{*}=A_{1} /\left(2 A_{2}\right)^{1 / 2}$ and the exponential integral $E i(-\infty)$. As $y_{*}$ is a quantity much larger than unity, being of order/ one can use the asymptotic expansions of these functions. The calculations are lengthy but straightforward. The values of the various integrals are given in Appendix A. The result is (retaining terms up to $\operatorname{order} A^{-6}$ ):

$$
\begin{aligned}
& S_{3 f}^{a}= \frac{m \mu}{2 \nu b^{2} A_{1}^{2}}\left[\frac{2}{A_{1}}\left(1-\frac{6 A_{2}}{A_{1}}\right)-\frac{3 a_{a}}{A_{1}^{2}}\left(1-\frac{10 A_{2}}{A_{1}^{2}}\right)\right. \\
&\left.+\frac{i U_{a}}{V_{0}}\left\{1-\frac{3 A_{2}}{A_{1}^{2}}-\frac{a_{a}}{A_{1}}\left(1-\frac{6 A_{2}}{A_{1}^{2}}\right)\right\}\right] .
\end{aligned}
$$

We now observe that $A_{1}$ can be written as

$$
A_{1}=a_{a}\left(1-\frac{i p_{a}}{2 \nu}\right) \text {, }
$$

where $p_{a}=v_{0} v_{a} / a_{a} b^{2}$ and $p_{a} / \nu<<1$. We can, therefore, again carry out the expansions of $A_{1}^{-n}$ in powers of $p_{a} / \nu$.

Retaining terms up to the fifth power in $p_{a} / \nu$ we obtain:

$-20-$ 


$$
\begin{aligned}
I_{i f}^{a} & =\frac{m \mu}{2(\pi)^{1 / 2} v_{0}^{3} b^{3} a_{a}^{2}}\left[\sum_{3} \frac{1}{a_{a}}\left(1-\frac{3 i U_{a}}{a_{a} v_{0}}-\frac{18}{a_{a}^{2}}\right)\right. \\
& +\sum_{5}\left\{\frac{3}{a_{a}}\left(i p_{a}+\frac{3 v_{0}^{2}}{a_{a}^{2} b^{2}}\right)-\frac{i U_{a}}{V_{0}}\left(-\frac{1}{2} i p_{a}+9 \frac{i}{a_{a}^{2}} p_{a}-\frac{3}{2} \frac{V_{0}^{2}}{a_{a}^{2} b^{2}}\right)\right\} \\
& \left.-\frac{3}{2} \sum_{7}\left\{\frac{3}{a_{a}} p_{a}^{2}+\frac{i U_{a}}{v_{0}}\left(\frac{1}{2} p_{a}^{2}-3 i p_{a} \frac{v_{0}^{2}}{a_{a}^{2} b^{2}}\right)\right\}-\frac{3}{4} \frac{U_{a}}{v_{0}} p_{a}^{3} \Sigma_{a}\right],
\end{aligned}
$$

where

$$
\Sigma_{m}=\int_{0}^{1} d y y^{m-1} \exp \left(-u_{a}^{2} y^{2} / 4 b^{2}\right) .
$$

It is, perhaps, worthwhile to remark again that the only assumption which has been made to evaluate the integral $I_{\text {/f }}^{a}$ is that the phase velocity of the wave is much larger than the mean thermal velocity; and this is indeed a legitimate one for the problem at hand.

We now assume that $m / M \ll 1$ and $v_{i}^{2} \ll<v_{0}^{2}$ which is quite valid in all cases of physical interest; the second requirement, as a matter of fact, is a consequence of the first one. Under the se assumptions, we have

$$
b^{2} \simeq \frac{1}{2} v_{0}^{2}, \quad p_{a} \simeq \frac{2 v_{a}}{a_{a} v_{0}}
$$

and

$$
\sum_{m}=\int_{0}^{1} d y y^{m-1} \exp \left(-U_{a}^{2} / 2 v_{0}^{2}\right) \text {. }
$$

The integrals $\Sigma_{m}$ have been evaluated numerically for suitable values of the parameter $A=V_{0}^{2} / U_{a}^{2}$. We thus finally obtain:

$-21-$ 


$$
\begin{aligned}
I_{1 f}^{a}= & -\frac{2 i k^{3}}{(2 \pi)^{1 / 2} V_{0}^{3}\left(\omega-k U_{a}\right)^{3}}\left[\sum_{3}\left\{1+\frac{3 k U_{a}}{\omega-k U_{a}}+\frac{18 k^{2} V_{0}^{2}}{\left(\omega-k U_{a}\right)^{2}}\right\}\right. \\
& -\Sigma_{5}\left\{\frac{U_{a}^{2}}{V_{0}^{2}}+\frac{9 k U_{a}}{\omega-k U_{a}}+\frac{18 k^{2} V_{0}^{2}}{\left(\omega-k U_{a}\right)^{2}}\left(1+U_{a}^{2} / V_{0}^{2}\right)\right\} \\
& \left.+\sum_{7} \frac{3 U_{a}^{2}}{v_{c}^{2}\left(\omega-k U_{a}\right)}\left\{k U_{a}+\frac{12 k^{2} v_{0}^{2}}{\left(\omega-k U_{a}\right)}\right\}-\sum_{q} \frac{6 U_{a}^{2}}{v_{0}^{2}} \frac{k^{2} U_{a}^{2}}{\left(\omega-k U_{a}\right)^{2}}\right],
\end{aligned}
$$

where we have put $\Delta=-i \omega f$ or the sake of convenience. Proceeding on similar lines, we obtain

$$
\begin{aligned}
I_{1 d}^{a}= & \frac{-2 i k^{3}}{(2 \pi)^{1 / k} v_{0}^{3}\left(\omega-k U_{a}\right)}\left[-\frac{8 k^{2} v_{0}^{2}}{\left(\omega-k v_{a}\right)} \Sigma_{3}\right. \\
& \left.+\sum_{5} 2 k v_{0}\left\{\frac{U_{a}}{v_{0}}+\frac{4 k v_{0}}{\left(\omega-k U_{a}\right)}\right\}-\sum_{7} \frac{8 k^{2} U_{a}^{2}}{\left(\omega-k v_{a}\right)}\right] .
\end{aligned}
$$

It is to be noted from Eq. (79) and (80) that in ion-electron collisions it is the frictional term which is the dominant one; the diffusion term tends to be smaller by a factor or order $1 / a_{a}$. Combining Eq. (79) and (80) we obtain for the contribution of the electron-ion collisions:

$$
\begin{aligned}
& I_{1}^{a}=-\frac{2 i k^{3}}{(2 \pi)^{1 / 2} V_{0}^{3}\left(\omega-k v_{a}\right)^{3}}\left[\Sigma_{3}\left\{1+\frac{3 k v_{a}}{\omega-k v_{a}}+\frac{10 k^{2} v_{0}^{2}}{\left(\omega-k v_{a}\right)^{2}}\right\}\right. \\
& -\sum_{5}\left\{\frac{U_{a}^{2}}{V_{0}^{2}}+\frac{7 k v_{a}}{\omega-k v_{a}}+\frac{10 k^{2} v_{0}^{2}}{\left(\omega-k V_{a}\right)^{2}}\left(1+\frac{q V_{a}^{2}}{5 V_{0}^{2}}\right)\right\} \\
& \left.+\sum_{7} \frac{U_{a}^{2}}{V_{0}^{2}\left(\omega-k v_{a}\right)}\left\{3 k v_{a}+\frac{28 k^{2} v_{0}^{2}}{\omega-k v_{a}}\right\}-\sum_{q} 6 \frac{V_{a}^{2}}{V_{0}^{2}} \frac{k^{2} V_{a}^{2}}{\left(\omega-k v_{a}\right)^{2}}\right] \text {. }
\end{aligned}
$$

Next we wish to evaluate

$$
I_{2 b f, d}^{a}=\int_{0}^{\infty} d \sigma \int d \eta e^{-\Delta\left(\sigma-\eta_{z}\right) / k} K_{2 f, d}^{b}(\sigma, \eta) P_{a z}(k, \eta),
$$

where

$$
K_{2 f}^{b}(\sigma, \eta)=\frac{1}{\pi^{2}} \frac{\sigma\left(\sigma-\eta_{z}\right)}{\left|\sigma_{m z}-\eta\right|^{2}} \exp \left\{-\frac{1}{2} \nu_{0}^{2}\left(\sigma e_{z}-\eta\right)^{2}-i U_{b}\left(\sigma-\eta_{z}\right)\right\}(83)
$$

and

$-22-$ 


$$
\left.K_{2 d}^{b}(\sigma, \eta)=-\frac{1}{2 \pi^{2}} \frac{[\sigma(\sigma-\eta z)]^{2}}{\left|\sigma e_{z}-\eta\right|^{4}} \exp \left\{-\frac{1}{2} v_{0}^{2}\left(\sigma_{m} e_{z}-\eta\right)\right)^{2}-i y_{b}\left(\sigma-\eta_{z}\right)\right\}(84)
$$

The evaluation of $I_{2 b f, d}^{a}$ proceeds on similar lines as $I_{\text {if }}^{a}$ and we find:

$$
\begin{aligned}
& I_{2 b f}^{a}=\frac{1}{(\pi)^{1 / 2} v_{0}^{6} a_{a}^{3}}\left[T_{3}\left(1-3 \frac{d}{a_{a}}+\frac{18}{a_{a}^{2}}\right)+T_{5}\left(\frac{9}{a^{2}}+\frac{9}{2} \frac{d}{a_{a}}\right.\right. \\
& \text { and } \left.\left.-\frac{1}{2} d^{2}-9 \frac{d^{2}}{a_{a}}\right)+T_{7} \frac{d^{2}}{a_{a}}\left(\frac{9}{a_{a}}+\frac{3}{4} d\right)+\frac{3}{4} \frac{d^{4}}{a_{a}^{2}} T_{9}\right] \\
& I_{2 b d}^{a}=\frac{1}{(\pi)^{1 / 2} v_{0}^{6} a_{a}^{3}}\left[\frac{8}{a_{a}^{2}} T_{3}-\frac{1}{a_{a}}\left(d+\frac{4}{a_{a}}\right) T_{5}-\frac{2 d^{2}}{a_{a}^{2}} T_{7}\right],(86)
\end{aligned}
$$

where

$$
d=i\left(u_{a}-u_{b}\right) / v_{0}, \quad a_{a}=\left(s+i k u_{a}\right) / k v_{0}
$$

and

$$
T_{m}=\int_{0}^{1} d y y^{m-1} e^{-y^{2}\left(U_{a}-u_{l}\right)^{2} / 4 v_{0}^{2}}
$$

Combining Eqs. (85) and (86), we obtain

$$
\begin{aligned}
I_{2 b}^{a} & =\frac{1}{(\pi)^{1 / 2} V_{0}^{6} a_{a}^{3}}\left[T_{3}\left(1-\frac{10}{a_{a}^{2}}-\frac{3 d}{a_{a}}\right)+T_{5}\left(\frac{5}{a_{a}^{2}}+\frac{7 d}{2 a_{a}}\right.\right. \\
& \left.\left.+\frac{1}{2} d^{2}-9 \frac{d^{2}}{a_{a}^{2}}\right)-T_{7} \frac{d^{2}}{a_{a}}\left(\frac{7}{a_{a}}+\frac{3}{4} d\right)+\frac{3}{4 a_{a}^{2}} d^{4} T_{9}\right] .
\end{aligned}
$$

In particular we find that for collisions between particles of the same stream, this reduces to

$$
I_{2 a}^{a}=\frac{1}{3(\pi)^{1 / 2} v_{0}^{6} a_{a}^{3}}\left(1-\frac{7}{a_{a}^{2}}\right) \text {. }
$$

$-23-$ 
Finally, we wish to evaluate

$$
I_{3 b f, d}^{a}=\int_{0}^{\infty} d \sigma \int d \eta e^{-\Delta(\sigma-\eta) / k} K_{3 f, d}^{b}(\sigma, \eta) P_{a z}(k, \eta),(91)
$$

where

$$
K_{3 f}^{b}(\sigma, \eta)=\frac{1}{\pi^{2}} \frac{\sigma_{\eta}}{\eta^{2}} \exp \left\{-\frac{1}{2} V_{0}^{2}\left(\sigma_{m} e_{z}-\eta\right)^{2}-i U_{f}\left(\sigma-\eta_{z}\right)\right\}(92)
$$

and

$$
K_{3 d}^{b}(\sigma, \eta)=-\frac{1}{2 \pi^{2}} \frac{\sigma^{2} \eta_{z}^{2}}{\eta^{4}} \exp \left\{-\frac{1}{2} v_{0}^{2}\left(\sigma_{m z}-\eta\right)^{2}-i u_{b}\left(\sigma-\eta_{z}\right)\right\} \text {. }
$$

On substituting for $P_{a z}(k, \eta)$ and $K_{3 f}^{b}(\sigma, \eta)$ into Eq. (91), we obtain:

$$
\begin{aligned}
& I_{3 b f}^{a}=-\frac{e^{\alpha_{a}^{2}}}{2 \pi^{2} v_{0}^{2}} \int_{0}^{\infty} d \sigma e^{-\Delta \sigma / k} \int d \eta\left[e^{-\eta_{+}^{2}}-2 \alpha_{a} E r f\left(\eta_{+}\right)\right] \text {(94) } \\
& \quad \times \frac{2 \sigma}{\eta^{2}} \eta_{z} \exp \left\{v_{0} a_{b} \eta_{z}-\frac{1}{2} v_{0}^{2} \eta_{z}^{2}-v_{0}^{2} \eta_{L}^{2}-\frac{1}{2} v_{0}^{2} \sigma_{+}^{2}+v_{0}^{2} \sigma \eta_{z}-i \sigma v_{b}\right\},
\end{aligned}
$$

where now it is convenient to define

$$
a_{a}=\left(\frac{A}{k}+i U_{a}\right) / v_{0}, \quad a_{b}=\left(\frac{p}{k}+i U_{b}\right) / v_{0}
$$

and

$$
(2)^{1 / 2} \eta_{+}=a_{a}+v_{0} \eta_{z} \text {. }
$$

We now first carry out the $\sigma$-integration. The result is

$$
\begin{aligned}
& I_{3 b f}^{a}=\frac{-e^{\alpha_{a}^{2}}}{\pi^{2} v_{0}^{4}} \int d \eta_{-} \frac{\eta_{z}}{\eta^{2}}\left\{1-2 \eta_{-} e^{\eta_{-}^{2}} \operatorname{Erf}\left(\eta_{-}\right)\right\} \\
& \quad \times\left\{e^{-\eta_{+}^{2}}-2 \alpha_{a} \operatorname{Erf}\left(\eta_{+}\right)\right\} \exp \left\{v_{0} a_{b} \eta_{z}-\frac{1}{2} v_{0}^{2} \eta_{z}^{2}-v_{0}^{2} \eta_{\perp}^{2}\right\},
\end{aligned}
$$

where

$$
(2)^{1 / 2} \eta_{-}=a_{b}-v_{0} \eta_{z}
$$

$-24-$ 
Again introducing a system of cylinderical co-ordinates in

space and carrying out the angular integration we get

$$
\begin{aligned}
I_{3 b f}^{a}=- & \frac{2 e^{\alpha_{a}^{2}}}{\pi v_{0}^{4}} \int_{-\infty}^{\infty} d \eta_{z}\left[e^{-\eta_{t}^{2}}-2 \alpha_{a} E r f\left(\eta_{t}\right)\right] \\
& \quad x \exp \left(a_{b} v_{0} \eta_{z}-\frac{1}{2} v_{0}^{2} \eta_{z}^{2}\right) S_{4 f},
\end{aligned}
$$

$$
S_{4 f}=\int_{0}^{\infty} d \eta_{1} e^{-v_{0}^{2} \eta_{1}^{2}} \frac{\eta_{L} \eta_{z}}{\left(\eta_{L}^{2}+\eta_{z}^{2}\right)}\left\{1-2 \eta_{-} e^{\eta^{2}} \operatorname{Erf}\left(\eta_{-}\right)\right\} .
$$

After some elementary reductions, Eq. (99) can be put in the form

$$
\begin{aligned}
S_{4 f}= & \int_{1}^{\infty} \frac{d \nu}{2 v_{0} \nu} e^{-(2-1) x^{2}}\left[x\left(a_{b}-x\right)(2)^{1 / 2}\right. \\
& \left.x e^{\left(a_{b}-x\right)^{2 / 2}} \operatorname{Erf}\left(\frac{a_{b}-x}{(2)^{1 / 2}}\right)-x\right],
\end{aligned}
$$

where we have put $x=v_{0} \eta_{z}$. On substituting this expression for $S_{4 f}$ into Eq. (98), we get

$$
I_{3 b f}^{a}=\frac{1}{\pi v_{0}^{6}} \int_{1}^{\infty} \frac{d \nu}{\nu} \int_{-\infty}^{\infty} d x e^{-\nu x^{2}-x d} F(x),
$$

where $d$ is defined in Eq. (87) and

$$
\begin{aligned}
F(x) & =\left[1-(2)^{1 / 2} a_{a} \exp \frac{1}{2}\left(a_{a}+x\right)^{2} \operatorname{Erf}\left(\frac{a_{a}+x}{(2)^{1 / 2}}\right)\right] \\
& \times\left[(2)^{1 / 2} x\left(a_{b}-x\right) e^{\left(a_{b}-x\right)^{2 / 2}} \operatorname{Erf}\left(\frac{a_{b}-x}{(2)^{1 / 2}}\right)-x\right] .
\end{aligned}
$$

If we carry out the asymptotic expansions of the error functions, we obtain on retaining terms unto $a^{-5}$ :

$$
F(x)=\frac{-x^{2}}{\left(a_{a}+x\right)\left(a_{b}-x\right)^{2}}+\frac{3 x^{2}}{\left(a_{a}+x\right)\left(a_{b}-x\right)^{4}}-\frac{a_{a} x}{\left(a_{a}+x\right)^{3}\left(a_{b}-x\right)^{2}} .
$$

$-25-$ 
Since $x / a \ll 1$, we can further expand the denominators in this expression in powers of $x / a$ and obtain:

$$
\begin{aligned}
F(x) & \simeq-\frac{x}{a_{a}^{2} a_{b}^{2}}+\frac{x^{2}}{a_{a} a_{b}^{2}}\left(-1+\frac{3}{a_{b}^{2}}+\frac{3}{a_{a}^{2}}-\frac{2}{a_{a} a_{b}}\right) \\
& +\frac{x^{3}}{a_{a} a_{l}^{2}}\left(\frac{1}{a_{a}}-\frac{2}{a_{b}}\right)+\frac{x^{4}}{a_{a} a_{b}^{2}}\left(-\frac{1}{a_{a}^{2}}+\frac{2}{a_{a} a_{b}}-\frac{3}{a_{l}^{2}}\right) .
\end{aligned}
$$

We now substitute this expression for $F(x)$ into $I_{3 b f}$ given by Eq. (101). The resulting integrations are then elementary and we obtain:

$$
\begin{aligned}
& I_{3 b f}^{a}=-\frac{2}{(\pi)^{1 / 2} v_{0}^{6} a_{a} a_{b}^{2}}\left[T_{3}\left(\frac{1}{2}+\frac{1}{a_{a} a_{b}}-\frac{3 k}{2 a_{a}^{2}}-\frac{3}{2 a_{b}^{2}}-\frac{d}{2 a}\right)\right. \\
& \quad+T_{5}\left\{\frac{3}{4}\left(\frac{1}{a_{a}^{2}}+\frac{3}{a_{b}^{2}}-\frac{2}{a_{a} a_{b}}\right)-\frac{3}{4} d\left(\frac{2}{a_{b}}-\frac{1}{a_{a}}\right)\right. \\
& \left.\quad+\left(\frac{d}{2}\right)^{2}\left(1-\frac{3}{a_{a}^{2}}-\frac{3}{a_{b}^{2}}+\frac{2}{a_{a} a_{b}}\right)\right\}+T_{7}\left\{3\left(\frac{d}{2}\right)^{2}\left(\frac{1}{a_{a}^{2}}+\frac{3}{a_{b}^{2}}-\frac{2}{a_{a} a_{b}}\right)\right. \\
& \left.\left.\quad-\left(\frac{d}{2}\right)^{3}\left(\frac{2}{a_{b}}-\frac{1}{a_{a}}\right)\right\}+\left(\frac{d}{2}\right)^{4}\left(\frac{1}{a_{a}^{2}}+\frac{3}{a_{b}^{2}}-\frac{2}{a_{a} a_{b}}\right) T_{9}\right] . \\
& I_{3 b d}=\frac{2}{(\pi)^{1 / 2} v_{0}^{6} a_{a} a_{b}^{3}}\left[\frac{1}{a_{a}} T_{3}+\left(\frac{3}{2 a_{b}}-\frac{1}{2 a_{a}}-\frac{d}{2}\right) T_{5}\right. \\
& \left.+\left(\frac{d}{2}\right)^{2}\left(\frac{3}{a_{b}}-\frac{1}{a_{a}}\right) T_{7}\right] \cdot
\end{aligned}
$$

On combining the foregoing expressions, we obtain:

$$
\begin{aligned}
I_{3 b}^{a}= & I_{3 b f}^{a}+I_{3 b d}^{a} \\
= & \frac{2}{(\pi)^{1 / 2} v_{0}^{6} a_{a} a_{b}^{2}}\left[T_{3}\left\{-\frac{1}{2}+\frac{3}{2}\left(\frac{1}{a_{a}^{2}}+\frac{1}{a_{b}^{2}}\right)+\frac{d}{2 a_{a}}\right\}\right. \\
& +T_{5}\left\{\frac{1}{a_{a} a_{b}}-\frac{3}{4}\left(\frac{1}{a_{a}^{2}}+\frac{1}{a_{b}^{2}}\right)+\frac{d}{2}\left(\frac{2}{a_{b}}-\frac{3}{2 a_{a}}\right)\right. \\
& \left.+\left(\frac{d}{2}\right)^{2}\left(-1+\frac{3}{a_{a}^{2}}+\frac{3}{a_{b}^{2}}-\frac{2}{a_{a} a_{b}}\right)\right\}+T_{7}\left(\frac{d}{2}\right)^{2}\left\{\left(\frac{5}{a_{a} a_{b}}-\frac{3}{a_{a}^{2}}-\frac{6}{a_{b}^{2}}\right)^{(107)}\right. \\
& \left.\left.-\frac{d}{2}\left(\frac{1}{a_{a}}-\frac{2}{a_{b}}\right)\right\}+T_{9}\left(\frac{d}{2}\right)^{4}\left(\frac{2}{a_{a} a_{b}}-\frac{1}{a_{a}^{2}}-\frac{3}{a_{b}^{2}}\right)\right] .
\end{aligned}
$$


In particular for collisions in the same stream, this reduces to

$$
I_{3 a}^{a}=-\frac{1}{3(\pi)^{1 / 2} v_{0}^{6} a_{a}^{3}}\left(1-\frac{27}{5 a_{a}^{2}}\right) .
$$

It is interesting to observe that for collisions in the same stream, the total contribution of the electron-electron collisions is

$$
I_{2 a}^{a}+I_{3 a}^{a}=-\frac{8}{15} \frac{1}{(\pi)^{1 / 2} v_{0}^{6} a_{a}^{5}}
$$

and compared with the electron-ion collisions, this is smaller by a factor $a_{a}^{-2}$. It turns out that the integral $T_{m}$ defined by Eq. (88) is about an order of magnitude smaller that the integral $\Sigma_{\mathrm{m}}$ defined by Eq. (78). Hence the contribution of the electronelectron collisions between one stream and the other is an order of magnitude smaller than the electron-ion collisions. Further, in electron-electron collisions, the diffusion terms tend to be of the same order as the frictional terms.

\section{ANALYSIS OF THE DISPERSION RELATION}

On carrying out the summations indicated in Eq. (47) the dispersion relation can be written as:

$$
\begin{gathered}
1=\frac{\omega_{k}^{2}}{k^{2}}\left[P_{1 z}(k, 0)+P_{2 z}(k, 0)+\frac{\nu_{c} v_{0}^{3}}{k}\left\{2 I_{1}^{\prime}+2 I_{1}^{2}+I_{21}^{2}(110)\right.\right. \\
\left.\left.+I_{21}^{\prime}+I_{22}^{2}+I_{22}^{\prime}+I_{31}^{\prime}+I_{32}^{2}+I_{31}^{2}+I_{32}^{\prime}\right\}\right], \\
-27 .
\end{gathered}
$$


where we shall now take $U_{1}=-U_{2}=U$. We introduce the following dimensionless variables:

$x=k U / \omega_{p}, y=\omega / \omega_{p}, A=v_{0}^{2} / U^{2}$ and $B=\nu_{c} / \omega_{p}$.

on using the foregoing relations, the dispersion relation can be written as:

$$
1=\frac{2\left(y^{2}+x^{2}\right)}{\left(y^{2}-x^{2}\right)^{2}}+C_{1}+C_{2}+C_{12}
$$

with

$$
\begin{gathered}
C_{1}=6 A x^{2} \frac{y^{4}+6 y^{2} x^{2}+x^{4}}{\left(y^{2}-x^{2}\right)^{4}} \\
C_{2}=-\frac{8 i B}{(2 \pi)^{1 / 2}} \frac{x^{2} y}{\left(y^{2}-x^{2}\right)^{3}}\left[f \frac{y^{2}+3 x^{2}}{x^{2}}+g \frac{y^{2}+x^{2}}{y^{2}-x^{2}}\right. \\
+h \frac{y^{4}+10 y^{2} x^{2}+5 x^{4}}{\left(y^{2}-x^{2}\right)^{2}}
\end{gathered}
$$

and

$$
\begin{aligned}
C_{12}= & -\frac{2 i B}{(\pi)^{1 / 2}} \frac{y}{\left(y^{2}-x^{2}\right)^{2}}\left[\frac { 1 } { y ^ { 2 } - x ^ { 2 } } \left\{\left(y^{2}+3 x^{2}\right) f_{1}\right.\right. \\
& \left.+A x^{2} \frac{y^{4}+10 y^{2} x^{2}+5 x^{4}}{\left(y^{2}-x^{2}\right)^{3}} g_{1}+4 x^{2} \frac{y^{2}+x^{2}}{\left(y^{2}-x^{2}\right)^{2}} l_{1}\right\} \\
& -2\left\{\frac{1}{2} T_{3}-T_{5} \frac{1}{A}+\frac{A x^{2}}{y^{2}-x^{2}} f_{2}-\frac{4 x^{2}}{y^{2}-x^{2}} g_{2}\right. \\
& \left.\left.+h_{2} \frac{A x^{2}\left(y^{2}+3 x^{2}\right)}{\left(y^{2}-x^{2}\right)^{2}}\right\}\right],
\end{aligned}
$$

$-28-$ 
where

$$
\begin{aligned}
& f=\Sigma_{3}-\frac{1}{A} \Sigma_{5}, \\
& g=4\left(3 \Sigma_{3}-7 \Sigma_{5}+\frac{3}{A} \Sigma_{7}\right),
\end{aligned}
$$$$
h=A\left\{\frac{2}{15}(2)^{1 / 2}+10\left(\Sigma_{3}-\Sigma_{5}\right)-\frac{2}{A}\left(9 \Sigma_{5}-14 \Sigma_{7}+\frac{3}{A} \Sigma_{9}\right)\right\},(118)
$$$$
f_{1}=T_{3}-\frac{2}{A} T_{5}
$$

$$
g_{1}=10 T_{3}-5 T_{5}-\frac{36}{A} T_{5}+\frac{28}{A} T_{7}-\frac{12}{A^{2}} T_{9}
$$$$
h_{1}=6 T_{3}-7 T_{5}+\frac{6}{A} T_{7}
$$

$$
f_{2}=\frac{3}{2} T_{3}+\frac{1}{4} T_{5}-\frac{1}{A} T_{5}-\frac{2}{A} T_{7}+\frac{1}{A^{2}} T_{9}
$$$$
g_{2}=T_{5}-\frac{1}{A} T_{7}
$$

and

$$
h_{2}=\frac{3}{2} T_{3}-\frac{3}{4} T_{5}-\frac{3}{A} T_{5}+\frac{6}{A} T_{7}-\frac{3}{A^{2}} T_{9} .
$$

$-29-$ 
It is worth noticing here that $A x^{2}=\left(k \lambda_{D}\right)^{2}$, a quantity which is always small compared with unity for all cases of physical interest. We shall now discuss some special cases of the dispersion relation (112).

a) $C_{1}=0, C_{2}=0, C_{12}=0$

In this case we obtain the well known cold case of two-stream instability which has been extensively discussed. The dispersion relation leads to the roots

$$
y^{2}=1+x^{2} \pm\left(1+4 x^{2}\right)^{1 / 2}
$$

The roots for $\omega$ are thus either real or purely imaginary; the latter one gives rise to instability which occurs for all values of $x$ which are less than $x_{c}=(2)^{1 / 2}$. It can be further seen that the growth rate of instability is maximum for $x=x_{\star}=(3)^{1 / 2} / 2$. The corresponding growth rate of maximum instability is $\left|y^{2}\right|=0.25$. Since the instability occurs only for the 'root with the negative sign in Eq. (78), we shall henceforth consider only this root and denote it by the subscript 'O'. i.e.

$$
y_{0}^{2}=1+x^{2}-\left(1+4 x^{2}\right)^{1 / 2}
$$

We now wish to see how $x_{c}$ and $x_{*}$ change when $A \neq 0$ and $B \neq 0$. b) $C_{2}=0, C_{12}=0, C_{1} \neq 0$.

In this case the dispersion relation reduces to

$$
1=\frac{2\left(y^{2}+x^{2}\right)}{\left(y^{2}-x^{2}\right)^{2}}+6 A x^{2} \frac{y^{4}+6 y^{2} x^{2}+x^{4}}{\left(y^{2}-x^{2}\right)^{4}} \text {. }
$$


This equation has been solved numerically for $y^{2}$ for given values of $x$ and $A$. The results are given in Table 1 and are plotted in Fig. 1 to illustrate the effect of thermal motions on two stream instability. It is rather interesting to note that in this case the maximum wavenumber $x_{c}^{(1)}$ upto which the instability occurs is larger than $\boldsymbol{x}_{\boldsymbol{c}}$ i.e.

$$
x_{c}^{(1)}>x_{c}=1.4142 .
$$

It is to be further noted that while the region of instability is increased, the growth rate of maximum instability is decreased, ie. $\left|y_{m}^{2}\right|<0.25$.

c) The effect of Collisions

In order to compute the effect of collisions, we first observe that the terms $C_{2}$ and $C_{12}$ are much smaller than unity so that we can solve Eq. (112) by iteration. Thus we can substitute $y=i y$ in $C_{2}$ and $C_{12}$ where $y_{1}$ is the solution of Eq. (127) and $y_{1}$ is real for $x \leqslant x_{c}^{(1)}$. Thus $C_{2}$ and $C_{12}$ become real in this region. Moreover $y_{1}$ is positive for an unstable mode which we are considering here. Since $C_{2}$ and $C_{12}$ are proportional toy and $y=0$ at $x=x_{c}$, we immediately conclude that the collisions cannot change $x_{c}$ and thus the region of instability. However to study the influence of collisions on the growth rate of maximum instability, we have solved Eq. (112) for various values of $A$ and $B$. The results are illustrated in Figures 2 and 3 .

It is rather interesting to find that the growth rate of maximum instability; which is reduced by the thermal motions of the particles, 
is enchanced by the collisions but the effect of thermal motions cannot be quenched by the Coulomb collisions.

V. CONCLUSIONS

In the absence of collisions, the region of instability of contrastreaming plasmas is increased by the thermal effects while the growth rate of maximum instability is decreased. However, on the assumption that the collisions are not too frequent $\left(\nu_{c} / \omega_{p}<<1\right)$ one finds that they have only second order effects. The region of instability is not affected by the presence of collisions while the growth rate of maximum instability is increased. The binary collisions that we have considered here do not seem to quench the effects of the thermal motions of the plasma species.

\section{ACKNOWLEDGEMENTS}

This work was partly carried out while B. B. held the Post-doctoral Resident Research Associateship and S. K. T. the Senior Post-doctoral Resident Research Associateship of the National Academy of Sciences, National Research Council. We are grateful to Dr. W. N. Hess for the facilities of the Laboratory for Theoretical studies of the Goddard Space Flight Center. We are deeply indebted to Mr. S. K. Malik and Mrs. E. Glover for assisting us with the numerical computations. 
APPENDIX

We wish to evaluate integrals of the type

$$
I_{m, n}=\int_{0}^{\infty} d x x^{m} \frac{\exp -\left(A_{1} x+\frac{1}{2} A_{2} x^{2}\right)}{\left(A_{1}+A_{2} x\right)^{n}} .
$$

These integrals can be readily evaluated in terms of the Error and the Exponential integrals defined by

$$
E r f(x)=\int_{x}^{\infty} e^{-y^{2}} d y
$$

and

$$
E_{i}(-x)=-\int_{x}^{\infty} \frac{e^{-y}}{y} d y \text {. }
$$

For large values of the argument, the functions have the asymptotic expansions (1<)

$$
E r f(x)=\frac{e^{-x^{2}}}{2 x}\left[1-\frac{1}{2 x^{2}}+\frac{3}{2^{2} x^{4}}-\frac{1.3 .5}{2^{3} x^{6}}+\cdots\right]
$$

and

$$
E_{i}(-x)=-\frac{e^{-x}}{x}\left[1-\frac{1}{x}+\frac{2 !}{x^{2}}-\frac{3 !}{x^{3}}+\cdots\right]
$$

After some elementary reductions, we find:

$$
\begin{aligned}
& I_{0,2}=\frac{1}{A_{1} A_{2}}\left[1-2 y_{*} e^{y_{*}^{2}} E r\left(y_{*}\right)\right], \\
& I_{0,3}=\frac{1}{2 A_{1}^{2} A_{2}}\left[1+y_{*}^{2} e^{y_{*}^{2}} E_{i}\left(-y_{*}^{2}\right)\right], \\
& I_{1,0}=\frac{1}{A_{2}}\left[1-2 y_{*} e^{y_{*}^{2}} E_{r} f\left(y_{*}\right)\right], \\
& I_{1,1}=\frac{e^{y_{*}^{2}}}{A_{1} A_{2}}\left[y _ { * } \left[E_{*}\left(y_{*}\right)+y_{*}\left[\left(-y_{*}^{2}\right)\right]\right.\right.
\end{aligned}
$$


and

$$
I_{2,0}=\frac{1}{A_{1} A_{2}} 2 y_{*}\left[-y_{*}+\left(1+2 y_{*}^{2}\right) e^{y_{*}^{2}} \operatorname{Erf}\left(y_{*}\right)\right],
$$

where $y_{*}=A_{1} /\left(2 A_{2}\right)^{1 / 2}$. These integrals have the asymptotic values:

$$
\begin{aligned}
& I_{0,2}=\frac{1}{A_{1}^{3}}\left(1-\frac{3 A_{2}}{A_{1}^{2}}+\frac{15 A_{2}^{2}}{A_{1}^{4}}-\cdots\right), \\
& I_{0,3}=\frac{1}{A_{1}^{4}}\left(1-\frac{4 A_{2}}{A_{1}^{2}}+\frac{24 A_{2}^{2}}{A_{1}^{4}}-\cdots\right) \\
& I_{1,0}=\frac{1}{A_{1}^{2}}\left(1-\frac{3 A_{2}}{A_{1}^{2}}+\frac{15 A_{2}^{2}}{A_{1}^{4}}-\cdots\right), \\
& I_{1,1}=\frac{1}{A_{1}^{3}}\left(1-\frac{5 A_{2}}{A_{1}^{2}}+\frac{33 A_{2}^{2}}{A_{1}^{4}} \cdots\right)
\end{aligned}
$$

and

$$
I_{2,0}=\frac{2}{A_{1}^{3}}\left(1-\frac{6 A_{2}}{A_{1}^{2}}+45 \frac{A_{2}^{2}}{A_{1}^{4}}-\cdots\right) .
$$




\section{REFERENCES}

1. F. D. Kahn, J. Fluid Mechanics 2, 601 (1957); Rev. Mod. Phys. 30, 1069 (1958)

2. E. N. Parker, Phys. Rev. 112, 1429 (1958)

3. J. D. Jackson, J. Nuclear Energy C, 1, 171 (1960)

4. D. A. Tidman \& G. Weiss, Proc. Symp. on Electromagnetics and Fluid Dynamics of Gaseous Plasma, Brooklyn Polytechnic Press, 111 (1962)

5. R. M. May, Phys. Fluids I, 1826 (1964)

6. E. P. Gross and M. Krook, Phys. Rev. 102, 593 (1956)

7. D. A. Tidman, Phys. Fluids 4 , 1379 (1961)

8. G. G. Comisar, Phys. Fluids $\underline{6}, 76$ (1963)

9. B. Buti and R. K. Jain, Phys. Fluids $\underline{8}, 2080$ (1965)

10. M. N. Rosenbluth, W. M. McDonald and D. L. Judd, Phys. Rev. 107, 1 (1957)

11. A. Lenard, Bul1. Am. Phys. Soc. 6, 189 (1961)

12. P. H. Rutherford and E. A. Frieman, Phys. Fluids 6, 1139, 1963.

13. E. Jahnke and F. Emde, Tables of higher functions, 6th Ed. (1960) 
TABLE 1

\begin{tabular}{|c|c|c|c|c|}
\hline A & $B$ & $x_{c}$ & $x_{*}$ & $-y^{2} *$ \\
\hline 0.0 & 0.0 & 1.414 & 0.866 & 0.250 \\
\hline 0.001 & $\begin{array}{l}0.000 \\
0.050 \\
0.075 \\
0.100\end{array}$ & $\begin{array}{l}1.416 \\
1.416 \\
1.416 \\
1.416\end{array}$ & $\begin{array}{l}0.867 \\
0.867 \\
0.867 \\
0.867\end{array}$ & $\begin{array}{l}0.249 \\
0.249 \\
0.249 \\
0.249\end{array}$ \\
\hline 0.01 & $\begin{array}{l}0.000 \\
0.050 \\
0.075 \\
0.100\end{array}$ & $\begin{array}{l}1.435 \\
1.435 \\
1.435 \\
1.435\end{array}$ & $\begin{array}{l}0.874 \\
0.874 \\
0.874 \\
0.874\end{array}$ & $\begin{array}{l}0.245 \\
0.245 \\
0.245 \\
0.245\end{array}$ \\
\hline 0.05 & $\begin{array}{l}0.000 \\
0.050 \\
0.075 \\
0.100\end{array}$ & $\begin{array}{l}1.516 \\
1.516 \\
1.516 \\
1.516\end{array}$ & $\begin{array}{l}0.915 \\
0.916 \\
0.917 \\
0.918\end{array}$ & $\begin{array}{l}0.227 \\
0.231 \\
0.232 \\
0.234\end{array}$ \\
\hline 0.10 & $\begin{array}{l}0.000 \\
0.050 \\
0.075 \\
0.100\end{array}$ & $\begin{array}{l}1.612 \\
1.612 \\
1.612 \\
1.612\end{array}$ & $\begin{array}{l}0.976 \\
0.982 \\
0.985 \\
0.988\end{array}$ & $\begin{array}{l}0.214 \\
0.225 \\
0.230 \\
0.235\end{array}$ \\
\hline
\end{tabular}




\section{Figure Captions.}

Figure 1. Effect of thermal motions on $x_{c}, x_{*}$ and $y^{2}$ for

$$
A=0.001,0.01,0.05 \text { and } 0.1 \text {. }
$$

Figure 2. Variation of $y^{2}$ with $x$ for $B=0$ and 0.1 is illustrated for $A=0.05$.

Figure 3. Variation of $y^{2}$ with $x$ for $B=0,0.05$ and 0.1 is illustrated for $\mathbf{A}=0.1$.

Title for Table 1

The values of $x_{c}, x_{*}$ and $y_{*}^{2}$ for various values of $A=v_{0}^{2} / U^{2}$, and $B=v_{c} / \omega_{p}$. 


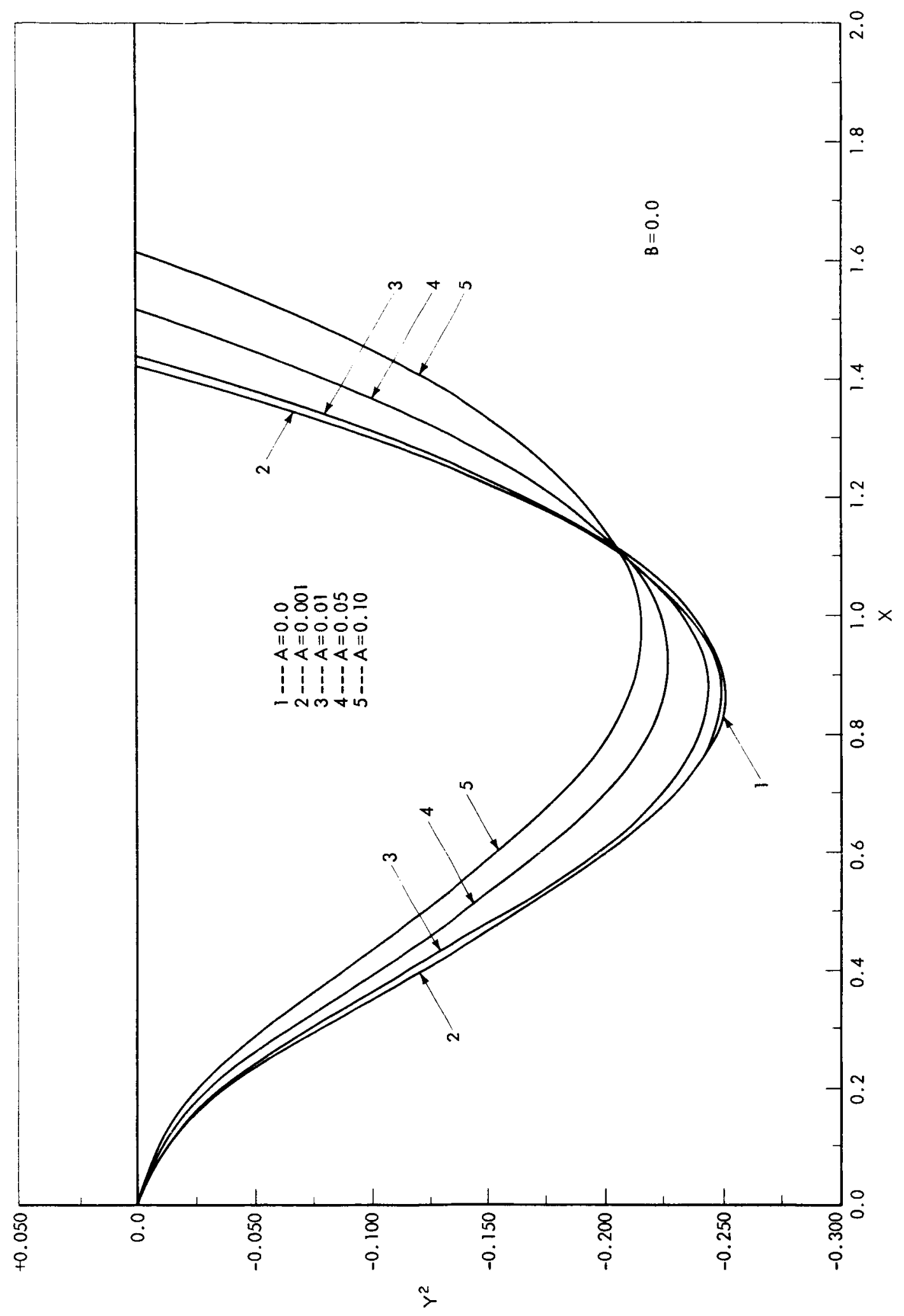




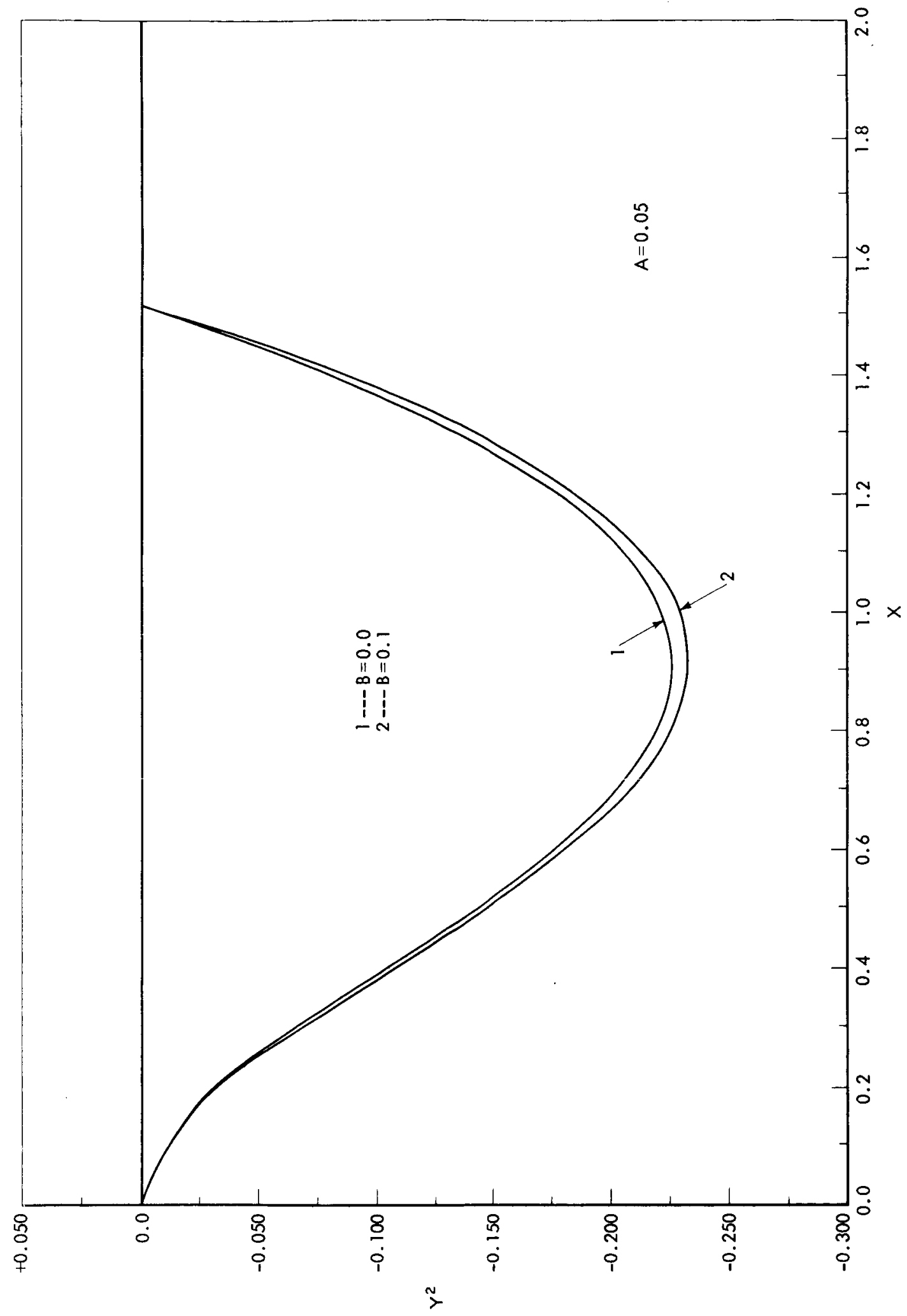




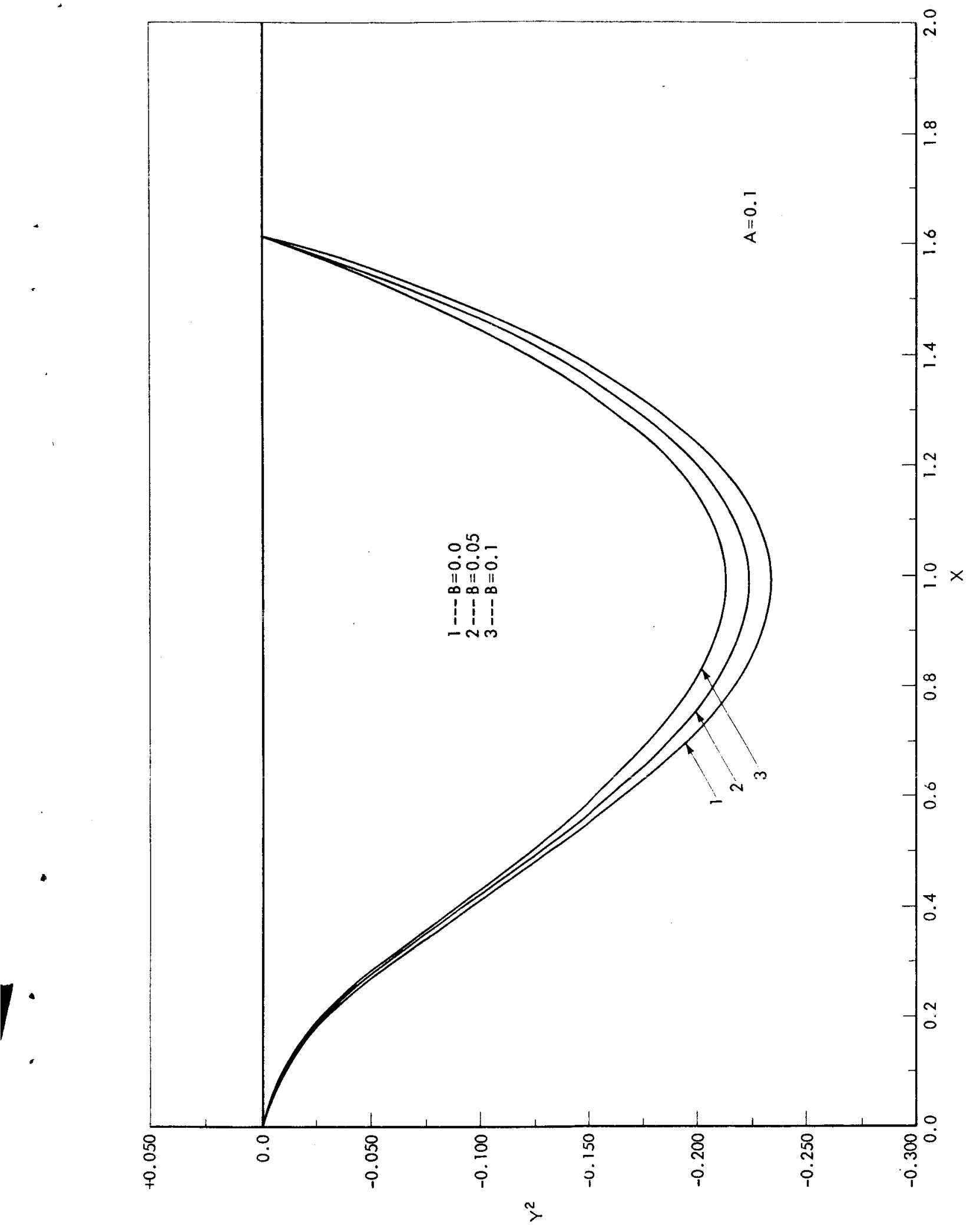

